\title{
Can Knee Joint Flexion Position of the Raised Lower Limb Affect Trunk Muscle Activation During Bird Dog Exercise in Subjects With Chronic Low Back Pain?
}

\author{
Kyung-ho Kim ${ }^{1,2}$, BPT, Chi-hun Lee ${ }^{2}$, PT, MSc, Seung-min Baik ${ }^{1,3}$, BPT, Heon-seock Cynn ${ }^{1}$, PT, PhD \\ ${ }^{1}$ Applied Kinesiology and Ergonomic Technology Laboratory, Department of Physical Therapy, The Graduate School, Yonsei University, \\ Wonju, '2Department of Rehabilitation Medicine, Seoul National University Bundang Hospital, Seongnam, ${ }^{3}$ Department of Physical Therapy, \\ Division of Health Science, Baekseok University, Cheonan, Korea
}

\author{
Article Info \\ Received December 29, 2021 \\ Revised January 18, 2022 \\ Accepted January 19, 2022 \\ Corresponding Author \\ Heon-seock Cynn \\ E-mail: cynn@yonsei.ac.kr \\ https://orcid.org/0000-0002-5810-2371
}

\section{Key Words}

Electromyography

Exercise

Low back pain
Background: Bird dog exercise (BDE) is one of the lumbar stabilization exercises that rehabilitate low back pain by co-contraction of the local and global muscles. Previous studies have reported the effect of various type of BDEs (for example, practicing the exercises on various surfaces and changing the limb movement) for muscle co-contraction.

Objects: This study aimed to investigate the effect of knee joint flexion position of the raised lower limb on abdominal and back muscle activity during BDE in patients with chronic low back pain (CLBP).

Methods: Thirteen males participated in this study (age: $32.54 \pm 4.48$ years, height: 177.38 $\pm 7.17 \mathrm{~cm}$ ). Surface electromyographic (SEMG) data of the internal abdominal oblique (IO), external abdominal oblique (EO), lumbar multifidus (MF), and thoracic part of the iliocostalis lumborum (ICLT) were collected in two knee joint flexion positions $\left(90^{\circ}\right.$ flexion versus $0^{\circ}$ flexion) during BDE. The SEMG data were expressed as a percentage of root mean square mean values obtained in the maximal voluntary isometric contraction.

Results: Greater muscle activity of the IO ( $p=0.001), M F(p=0.009)$, and ICLT ( $p=0.021)$ of the raised lower limb side and the EO ( $p=0.001)$ and MF ( $p=0.009)$ of the contralateral side were demonstrated in the knee joint flexion position compared to the knee joint extension position. Greater local/global activity ratios of the abdominal muscle (i.e., IO and EO) of the raised lower limb ( $p=0.002$ ) and the back muscle (i.e., MF and ICLT) of the contralateral side $(p=0.028)$ were also noted in the knee joint flexion position.

Conclusion: BDE with a knee joint flexion position might be recommended as an alternative lumbar stabilization exercise to enhance muscle activity in both the raised lower limb and the contralateral sides of the trunk for individuals with CLBP.

\section{INTRODUCTION}

Low back pain (LBP) is a frequent diagnosis and high-cost musculoskeletal disease in today, with up to $70 \%-80 \%$ of the population showing symptoms at least once during their lifetime [1,2]. Patients with LBP may obtain some benefits through an appropriate level of exercise $[3,4]$.

Lumbar stabilization exercises (LSEs) are an exercise for cocontraction of the local and global muscles for lumbar stability [5]. To achieve a level of spinal stability for both preventing and rehabilitating LBP, the co-contraction of several trunk muscles is considered necessary [6,7]. LSE is used to increase spinal stability in various postures, including prone, supine, sitting, standing, side bridge, or quadruped [8-13]. Practically, the LSE intensity is generally adjusted by modifying the exercise difficulty through changes in the exercise method (for example, changing the base of support and/or the lever arm and practicing the exercises on various devices/surfaces) [1419]. Bird dog exercise (BDE) in a quadruped position is one of the most commonly used LSEs [14,16,17,20,21]. BDE consists of different limb movements in a quadruped posture with the spine fixed in a neutral position [12,22-24]. BDE performed on unstable surfaces (for example, gym balls, hemisphere balls, and slings) increases muscle co-contraction [24-26]. BDEs with the movements of raised limbs increase demands of core stability and muscle co-contraction [24,27]. 
The inferior fibers of the internal abdominal oblique (IO) are considered to represent local muscle activity, while the back muscles, lumbar multifidus (MF), and lumbar part of the iliocostalis lumborum are the so-called local system because of their origin or insertion at the vertebrae or maintaining lumbar stability $[28,29]$. The rectus abdominis (RA), external abdominal oblique (EO), and thoracic part of the iliocostalis lumborum (ICLT) transfer the load between the pelvis and the thoracic rib cage, and are called global system [28,29]. In particular, great emphasis has been placed on facilitating local system muscles more than global system muscles in patients with chronic low back pain (CLBP) [30-34].

No previous study has examined the influence of the knee joint flexion position of the raised lower limb during BDE in subjects with CLBP. Thus, the purpose of this study was to compare the trunk muscle activity of two knee joint flexion positions $\left(90^{\circ}\right.$ flexion and $0^{\circ}$ flexion) on trunk muscle activity during BDE in subjects with CLBP. We hypothesized that there would be differences in trunk muscle activity between the two knee joint flexion positions.

\section{MATERIALS AND METHODS}

\section{Subjects}

G-power software (ver. 3.1.6; Franz Faul, Kiel University, Kiel, Germany) was used to conduct the power analyses. The necessary sample size of 13 subjects, obtained by a previous pilot study of five participants, was calculated to achieve a power of 0.80 and an effect size of 0.75 , with an alpha level of 0.05. The subjects included 13 male patients with CLBP. The demographics (i.e., age, height, weight, body mass index, duration of LBP, Oswestry Low Back Pain Disability Index (ODI), and back pain intensity) of the subjects with CLBP are summarized in Table 1.

The inclusion criterion was subjects with nonspecific LBP for more than three months without radiating pain and disk prolapse [35]. The exclusion criteria were as follows: taking medication for LBP; history of back surgery; had experienced thoracic and cervical pain; or had other causes of back pain, demonstrated hip flexor shortness, as assessed by the Thomas test, or had rectus femoris shortness, as assessed by Ely's test. Before participation, all subjects were acquainted with the procedure of the experiment and signed informed consent forms. This study was approved by Institutional Review Board
Table 1. Demographics of subjects with chronic low back pain $(N=13)$

\begin{tabular}{lc}
\hline \multicolumn{1}{c}{ Variable } & Value \\
\hline Age $(\mathrm{y})$ & $32.5 \pm 4.5$ \\
Height $(\mathrm{cm})$ & $177.4 \pm 7.2$ \\
Weight $(\mathrm{kg})$ & $75.5 \pm 14.3$ \\
BMI $\left(\mathrm{kg} / \mathrm{m}^{2}\right)$ & $23.9 \pm 3.2$ \\
Duration of LBP (mo) & $23.9 \pm 11.3$ \\
ODI (\%) & $12.8 \pm 7.6$ \\
Back pain intensity (NRS) & $2.7 \pm 1.4$ \\
\hline
\end{tabular}

Values are presented as mean \pm standard deviation. BMI, body mass index; LBP, low back pain; ODI, Oswestry Disability Index; NRS, numeric rating scale.

of the Graduate School, Yonsei University, Wonju (IRB no. 1041849-202104-BM-059-02).

\section{Pain Assessment and Electromyography Technique}

The average intensity of LBP was measured using a numeric rating scale (NRS) anchored with "no pain" and "the worst possible pain imaginable". The reliability and validity of NRS are supported by evidence across many populations [36,37]. The severity of LBP was scored using the ODI, a 10-point patient-reported questionnaire most commonly used to measure disability, quality of life, and impairment caused by LBP [38].

Signals from surface electromyography (SEMG) were recorded by a Noraxon TeleMyo-DTS (Noraxon Inc, Scottsdale, AZ, USA) and were analyzed using MyoResearch Master Edition software package (ver. 3.16; Noraxon Inc, Scottsdale, AZ, USA). Myoelectrical signals from both sides of an EO, IO, ICLT, and MF muscles were digitized, amplified (gain $=300$ ), and sampled at $1,500 \mathrm{~Hz}$ with an $\mathrm{A} / \mathrm{D}$ resolution system of 16 bit (EMGworks acquisition; Delsys Inc, Boston, MA, USA). A band pass filter was used between $20-400 \mathrm{~Hz}$, and the root mean square was calculated.

Before electrode placement, the skin was shaved and rubbed with an alcohol swab. Disposable surface $\mathrm{Ag} / \mathrm{AgCl}$ electrodes were placed bilaterally over each muscle according to guidelines [39]. To minimize crosstalk from multilayer muscles, a 1 $\mathrm{cm}$ diameter electrode was selected. Center-to-center interelectrode distance was $2.5 \mathrm{~cm}$. Electrode pairs were placed parallel to the muscle fibers over the following muscles according to the Surface ElectroMyoGraphy for the Non-Invasive Assessment of Muscles (SENIAM) recommendations: the bilateral EO, $15 \mathrm{~cm}$ lateral to the umbilicus; bilateral IO, at the midway between the anterior iliac spine and symphysis pubis, above the inguinal ligament; bilateral ICLT, above and below the L1 
level, midway between the midline and the lateral aspect of the body; and bilateral MF, lateral to the midline of the body, above and below a line connecting both posterior superior iliac spine. For normalization of the SEMG data, three maximal voluntary isometric contractions (MVIC) were performed for each muscle according to the manual muscle testing positions [40].

Five seconds of the MVIC were collected, with maximal effort against manual resistance, and a 2-minute of rest intervals were given between contractions [41]. SEMG data segments from one to four seconds on each MVIC trial were selected for assessing average electromyographic amplitudes [42,43]. The mean of the three seconds over three trials was calculated for data analysis. The gathered SEMG amplitudes for each muscle were reported as a percentage of root mean square mean values obtained in MVIC (\%MVIC).

\section{Procedures}

The subjects performed 20 minutes familiarization sessions for two knee joint flexion positions during the BDE until the appropriate movements were performed. After the session of familiarization, the subjects had 15 minutes of rest to avoid muscle fatigue [12]. The two knee joint flexion positions of the BDE were performed three times each, with a 1 minute rest period in between. The order of the exercises was randomized for each subject to reduce possible effects.

\section{1) Bird dog exercise with knee joint $0^{\circ}$ flexion position}

The subjects maintained a quadruped position and raised the lower limb of the painful low back side (i.e., area of usual pain) with a fully extended knee joint and the contralateral

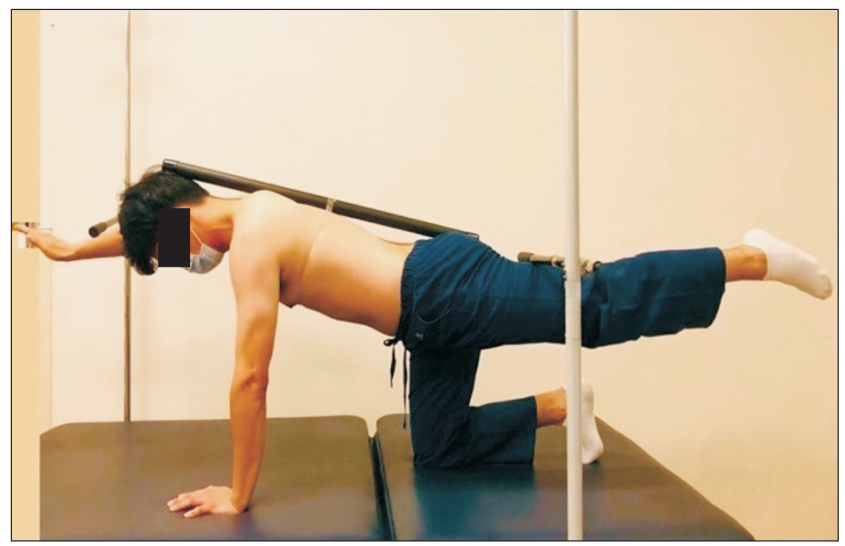

Figure 1. Bird dog exercise with a $0^{\circ}$ knee flexion position. arm, and those with bilateral LBP raised the lower limb of the more painful low back side while balancing the quadruped position [44]. Two target bars were used to confirm the horizontal position of the raised arm and leg. The target bars were set at the subject's raised wrist and the middle of the thigh. In addition, the subjects kept their supporting arms and thighs parallel to avoid leaning of the body (Figure 1).

\section{2) Bird dog exercise with knee joint $90^{\circ}$ flexion position} Bird dog exercise with knee joint $90^{\circ}$ flexion position (BDKFE) was performed in the same way as BDE, except that the knee joint was at $90^{\circ}$ flexion. The subjects raised the painful lower limb with the knee joint at $90^{\circ}$ flexion (measured using a goniometer). To confirm the maintenance of $90^{\circ}$ of knee joint flexion of the raised lower limb, a target bar was set at the raised middle calf of each subject. The other two target bars were positioned in the same position as the BDE. When knee joint flexion of $90^{\circ}$ was not sustained (i.e., the subject was unable to maintain the light contact of the calf to the target bar), the principal investigator (PI) provided verbal feedback so that the posture could be modified as in the exercise protocol. In addition, to prevent any uncontrolled lumbopelvic motion (i.e., anterior pelvic tilt associated with lumbar hyperextension, hip hiking/pelvic drop, or pelvic rotation) that might occur during BDE and BDKFE, the guide bar was longitudinally fixed using Scotch Tape (3M Inc, Saint Paul, MN, USA) and the aforementioned lumbopelvic motions were carefully monitored by the PI (Figure 2).

\section{Statistical Analysis}

SPSS (ver. 26.0; IBM Co., Armonk, NY, USA) program was

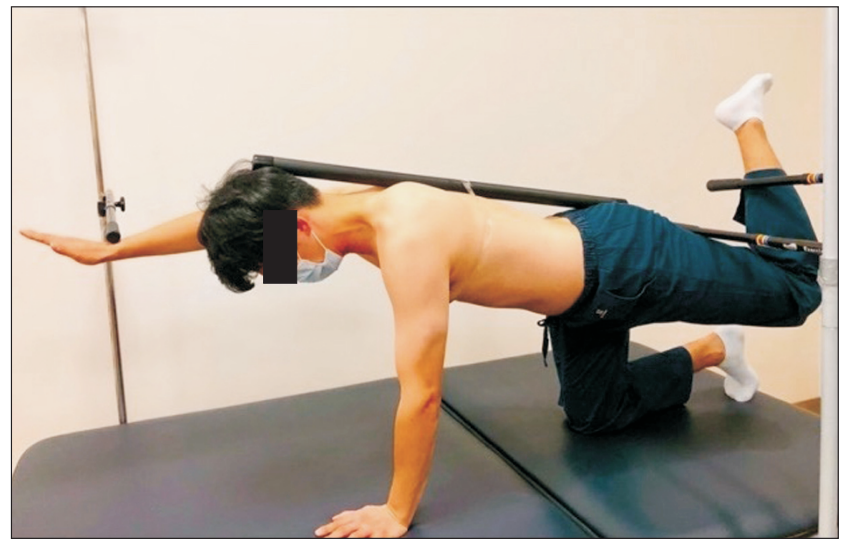

Figure 2. Bird dog exercise with a $90^{\circ}$ knee flexion position. 
used for statistical analyses. The Kolmogorov-Smirnov Z-test was used to confirm whether the continuous data had a normal distribution. Given that the collected data were not normally distributed, the Wilcoxon signed-rank test was performed to measure the differences in the normalized SEMG activity of the IO, EO, MF, and ICLT, and the local/global ratios of abdominal muscle activity (IO/EO) and back muscle activity (MF/ICLT) between different knee flexion positions during quadruped stabilization exercises (i.e., BDE and BDKFE). Significance was set at an alpha level of 0.05 for all analyses.

\section{RESULTS}

\section{IO, EO, MF, and ICLT Muscle Activity}

Greater muscle activity of the IO ( $p=0.001)$ MF ( $p=0.009)$,

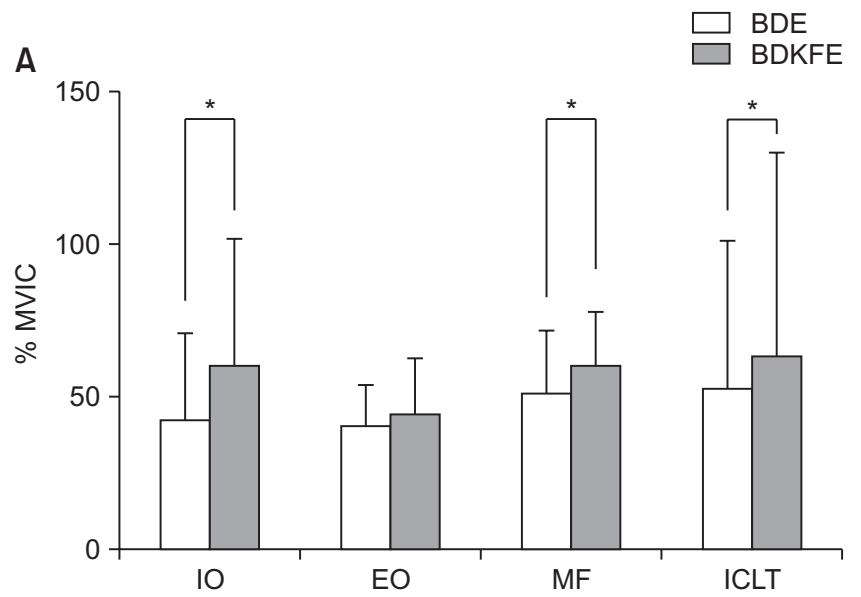

and ICLT ( $p=0.021)$ of the raised lower limb and the EO ( $p=$ 0.001) and MF ( $\mathrm{p}=0.009)$ of the contralateral side were shown in patients who performed BDKFE compared to those who performed BDE. There were no significant differences in the muscle activity of the EO of the raised lower limb and the IO and ICLT of the contralateral side between BDE and BDKFE (Figure 3).

\section{IO/EO and MF/ICLT Muscle Activity Ratios}

The local/global activity ratios of the back muscle of the raised lower limb and the abdominal muscle of the contralateral side were not significantly different between BDE and BDKFE. The local/global activity ratios of the abdominal muscle of the raised lower limb (IO/EO, p = 0.002) and the back muscle of the contralateral side (MF/ICLT, $\mathrm{p}=0.028$ ) were

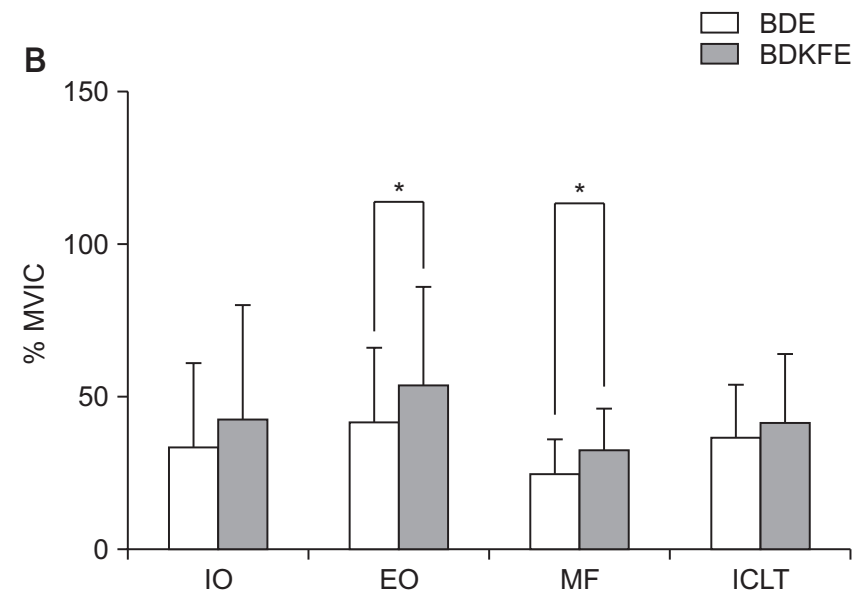

Figure 3. Comparison of trunk muscle activation during two knee joint flexion positions in bird dog exercise. (A) Raised lower limb. (B) Contralateral side. $\mathrm{BDE}$, bird dog exercise with knee joint $0^{\circ}$ flexion position; BDKFE, bird dog exercise with knee joint $90^{\circ}$ flexion position; 10 , internal abdominal oblique; EO, external abdominal oblique; MF, lumbar multifidus; ICLT, thoracic part of the iliocostalis lumborum; MVIC, maximal voluntary isometric contractions. ${ }^{*} p<0.05$
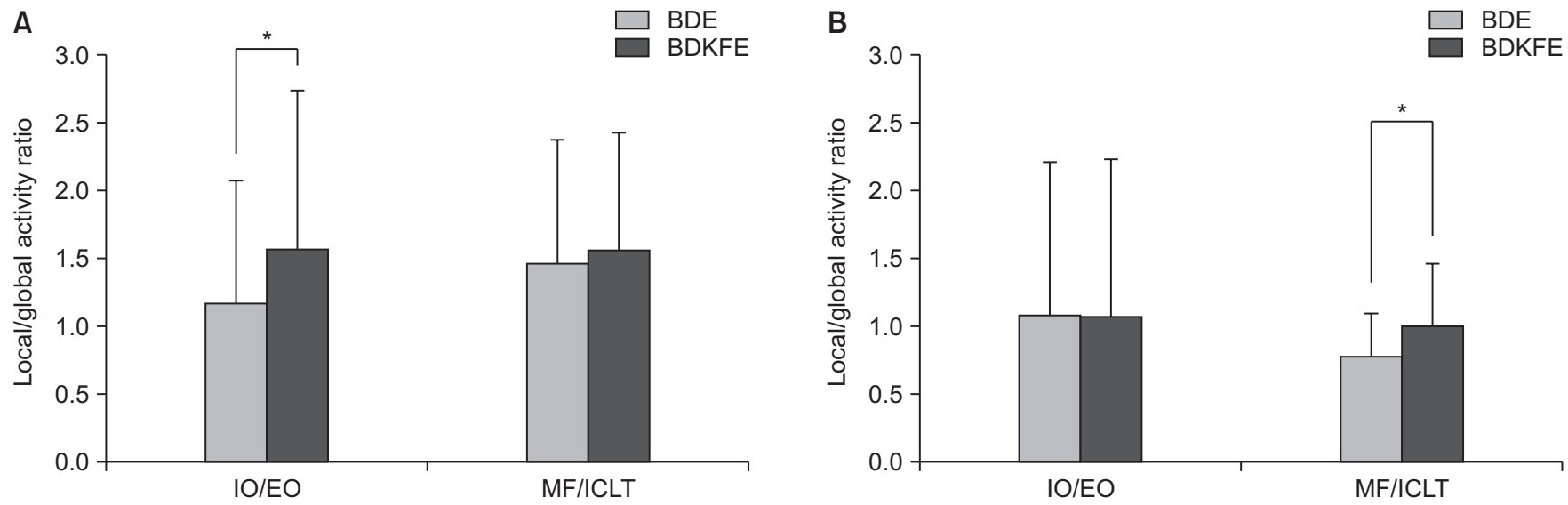

Figure 4. Local/global activity ratio of abdominal back muscles during two knee joint flexion positions in bird dog exercise. (A) Raised lower limb. (B) Contralateral side. BDE, bird dog exercise with knee joint $0^{\circ}$ flexion position; BDKFE, bird dog with knee flexion $90^{\circ}$ exercise; 10 , internal abdominal oblique; EO, external abdominal oblique; MF, lumbar multifidus; ICLT, thoracic part of the iliocostalis lumborum. *p $<0.05$. 
significantly different between BDE and BDKFE (Figure 4).

\section{DISCUSSION}

The purpose of this study was to evaluate the trunk muscle activities and ratios during BDE and BDKFE, in patients with CLBP. To our knowledge, this is the first study to examine the effect of knee joint flexion on both the raised and contralateral sides of trunk muscle activity in subjects with CLBP. Significantly greater muscle activity of the IO, MF, and ICLT of the raised lower limb and $\mathrm{EO}$ and MF of the contralateral side were noted during BDKFE compared to BDE. These findings support our research hypothesis.

In the present study, the significantly greater IO muscle activity (42\%) of the raised lower limb and MF muscle activity (raised lower limb by $17 \%$, contralateral side by 32\%) of both sides were found in response to BDKFE compared to BDE. Previous studies have reported conflicting findings $[5,8]$. These results differ from previous studies of healthy subjects, in which greater activity of the global muscles was shown when BDE was performed on an unstable surface (i.e., a more difficult level). However, the activity of the local muscles was not different between stable and unstable settings [5]. In the study by Souza et al. [8], there were no significant differences according to the level of exercise in abdominal muscles. These differences may be due to the presence or absence of a pathological state. In this study, we found greater muscle activity during BDKFE than during BDE in subjects with CLBP, especially for the local trunk muscles, IO, and MF. This result may imply that a higher contribution of the local muscles was required for lumbar segmental stabilization and intra-abdominal pressure during BDKFE compared to BDE in subjects with CLBP. In addition, it is believed that segmental control of the IO of the raised lower limb was required as a counterforce to offset the additional load to lumbar lordosis during BDKFE. Greater EO activity of the contralateral side was observed during BDKFE than during BDE. This is consistent with the results of a previous study in which an unstable surface increased the need to control excessive trunk rotation, following which, the EO activity was increased to maintain a quadruped position during BDKFE (i.e., to counter excessive rotation) [5].

In addition, significantly greater muscle activity was found for the ICLT of the raised lower limb during BDKFE compared to BDE. A previous study suggested that enhanced local muscle activation combined with the global muscle is important [5]. This finding can be attributed to the increased complexity caused by the addition of knee flexion to the BDE. Increased complexity may have led to trunk instability, so an increased level of coactivation between local and global muscles (i.e., ICLT in our study) were necessary.

A significantly greater IO/EO muscle activity ratio (34\%) of the raised lower limb and MF/ICLT muscle activity ratio (28\%) of the contralateral side were observed during BDKFE compared to BDE. The findings of the current study are in accordance with those of previous studies that demonstrated enhanced local muscle to global muscle ratio in subjects with CLBP by high levels of exercise [45]. However, several previous studies with healthy individuals reported higher muscle activity in the global muscles during high levels of exercise [5,27,46].

Another study reported no significant difference in the local muscle activity ratio between stabilization exercises performed on a stable and unstable surface [47]. These results showed that not only the co-contraction of local and global muscles but also the activation of local muscles was increased during BDKFE compared to BDE in subjects with CLBP. These findings suggest that the relative demand in the local muscle group increased as the level of exercise was higher in CLBP patients with weak local muscle groups.

In this study, the increase in MF activity on both sides during BDKFE was notable. A previous study of patients with CLBP showed that dysfunction and atrophy of the MF could allow spinal instability and may be predominant factors contributing to the recurrence of CLBP. Therefore, selective strengthening of the MF could be essential for the rehabilitation of CLBP [31]. Kim et al. [48] found that leveled LSEs may be an useful way to train the lumbar stabilizing muscles in individuals with LBP. The findings of our study support the need for graded LSEs. However, the clinical application of BDKFE in individuals with CLBP should be applied gradually based on the ability of the patients to maintain posture. These findings could be a guide for physical therapists when they make effective prescriptions for LSEs based on the patient's progression.

The present study also has several limitations. First, it includes only young adult male participants with CLBP. The reason was to minimize the effects of confounding variables, such as age, sex, flexibility, and muscle mass. However, this restriction limits the generalizability of the results to all patients. Second, the lumbar lordosis of the both exercises was 
not specifically measured using kinematic data. Third, other global muscles, including the RA muscle, were not monitored during the two exercises. Fourth, pain may have interfered with maximum effort and reduced its reliability during MVIC trials in CLBP patients. Fifth, this study did not consider the variables such as the dominant lower limb side and painful low back side that may have affected the results.

Finally, the results can be limitedly generalized only to individuals with low levels of disability, as the average ODI was $12.8 \pm$ 7.6. Further research is necessary to include female subjects, and those with a wider range of ages and levels of disability. Furthermore, a longitudinal studies are needed to investigate the long-term effects of BDKFE compared to BDE.

\section{CONCLUSIONS}

We conclude that BDKFE might be recommended for individuals with CLBP as a more intensive lumbar stabilization exercise than BDE. Clinically, these findings may have implications for the exercise selection, suggesting that performing BDKFE may be a way to induce greater trunk muscle activity in subjects with CLBP.

\section{CONFLICTS OF INTEREST}

No potential conflict of interest relevant to this article was reported.

\section{AUTHOR CONTRIBUTIONS}

Conceptualization: KK, HC. Data curation: KK, CL. Formal analysis: KK, CL, SB. Investigation: KK, CL, SB, HC. Methodology: KK, SB, HC. Project administration: KK, CL, HC. Resources: KK, CL, HC. Supervision: KK, HC. Validation: HC. Visualization: KK, CL. Writing - original draft: KK. Writing - review \& editing: KK, HC.

\section{ORCID}

Kyung-ho Kim, https://orcid.org/0000-0002-4148-7439

Chi-hun Lee, https://orcid.org/0000-0001-5948-9240

Seung-min Baik, https://orcid.org/0000-0001-7885-9374

\section{REFERENCES}

1. van Tulder M, Koes B. Low back pain and sciatica: chronic. Clin Evid 2002;(7):1032-48.

2. Ekman M, Jönhagen S, Hunsche E, Jönsson L. Burden of illness of chronic low back pain in Sweden: a cross-sectional, retrospective study in primary care setting. Spine (Phila Pa 1976) 2005;30(15):1777-85.

3. Hides JA, Stokes MJ, Saide M, Jull GA, Cooper DH. Evidence of lumbar multifidus muscle wasting ipsilateral to symptoms in patients with acute/subacute low back pain. Spine (Phila Pa 1976) 1994;19(2):165-72.

4. Ekstrom RA, Donatelli RA, Carp KC. Electromyographic analysis of core trunk, hip, and thigh muscles during 9 rehabilitation exercises. J Orthop Sports Phys Ther 2007;37(12):75462.

5. Imai A, Kaneoka K, Okubo Y, Shiina I, Tatsumura M, Izumi S, et al. Trunk muscle activity during lumbar stabilization exercises on both a stable and unstable surface. J Orthop Sports Phys Ther 2010;40(6):369-75.

6. Axler CT, McGill SM. Low back loads over a variety of abdominal exercises: searching for the safest abdominal challenge. Med Sci Sports Exerc 1997;29(6):804-11.

7. Butcher SJ, Craven BR, Chilibeck PD, Spink KS, Grona SL, Sprigings EJ. The effect of trunk stability training on vertical takeoff velocity. J Orthop Sports Phys Ther 2007;37(5):223-31.

8. Souza GM, Baker LL, Powers CM. Electromyographic activity of selected trunk muscles during dynamic spine stabilization exercises. Arch Phys Med Rehabil 2001;82(11):1551-7.

9. Kavcic N, Grenier S, McGill SM. Quantifying tissue loads and spine stability while performing commonly prescribed low back stabilization exercises. Spine (Phila Pa 1976) 2004;29(20):2319-29.

10. Stevens VK, Coorevits PL, Bouche KG, Mahieu NN, Vanderstraeten GG, Danneels LA. The influence of specific training on trunk muscle recruitment patterns in healthy subjects during stabilization exercises. Man Ther 2007;12(3):271-9.

11. Liebenson C, Karpowicz AM, Brown SH, Howarth SJ, McGill $\mathrm{SM}$. The active straight leg raise test and lumbar spine stability. PM R 2009;1 (6):530-5.

12. García-Vaquero MP, Moreside JM, Brontons-Gil E, PecoGonzález N, Vera-Garcia FJ. Trunk muscle activation during stabilization exercises with single and double leg support. J Electromyogr Kinesiol 2012;22(3):398-406. 
13. Rabin A, Shashua A, Pizem K, Dar G. The interrater reliability of physical examination tests that may predict the outcome or suggest the need for lumbar stabilization exercises. J Orthop Sports Phys Ther 2013;43(2):83-90.

14. Boucher JA, Preuss R, Henry SM, Dumas JP, Larivière C. The effects of an 8-week stabilization exercise program on lumbar movement sense in patients with low back pain. BMC Musculoskelet Disord 2016;17:23.

15. Chuter VH, de Jonge XA, Thompson BM, Callister R. The efficacy of a supervised and a home-based core strengthening programme in adults with poor core stability: a three-arm randomised controlled trial. Br J Sports Med 2015;49(6):3959.

16. El Shemy SA. Trunk endurance and gait changes after core stability training in children with hemiplegic cerebral palsy: a randomized controlled trial. J Back Musculoskelet Rehabil 2018;31 (6):1159-67.

17. Hoglund LT, Pontiggia L, Kelly JD 4th. A 6-week hip muscle strengthening and lumbopelvic-hip core stabilization program to improve pain, function, and quality of life in persons with patellofemoral osteoarthritis: a feasibility pilot study. Pilot Feasibility Stud 2018;4:70.

18. Mills JD, Taunton JE, Mills WA. The effect of a 10-week training regimen on lumbo-pelvic stability and athletic performance in female athletes: a randomized-controlled trial. Phys Ther Sport 2005;6(2):60-6.

19. Parkhouse KL, Ball N. Influence of dynamic versus static core exercises on performance in field based fitness tests. J Bodyw Mov Ther 2011;15(4):517-24.

20. Toprak Çelenay Ş, Özer Kaya D. An 8-week thoracic spine stabilization exercise program improves postural back pain, spine alignment, postural sway, and core endurance in university students: a randomized controlled study. Turk J Med Sci 2017;47(2):504-13.

21. Watson T, Graning J, McPherson S, Carter E, Edwards J, Melcher I, et al. Dance, balance and core muscle performance measures are improved following a 9-week core stabilization training program among competitive collegiate dancers. Int J Sports Phys Ther 2017;12(1):25-41.

22. Bjerkefors A, Ekblom MM, Josefsson K, Thorstensson A. Deep and superficial abdominal muscle activation during trunk stabilization exercises with and without instruction to hollow. Man Ther 2010;15(5):502-7.

23. Vera-García FJ, Barbado D, Moreno-Pérez V, Hernández-Sán- chez S, Juan-Recio C, Elvira JLL. Core stability: evaluation and training criteria. Rev Andal Med Deport 2015;8(3):130-7.

24. Vera-Garcia FJ, Barbado D, Moya M. Trunk stabilization exercises for healthy individuals. Rev Bras Cineantropom Desempenho Hum 2014;16(2):200-11.

25. Calatayud J, Casaña J, Martín F, Jakobsen MD, Colado JC, Andersen LL. Progression of core stability exercises based on the extent of muscle activity. Am J Phys Med Rehabil 2017;96(10):694-9.

26. Czaprowski D, Afeltowicz A, Gębicka A, Pawłowska P, Kędra A, Barrios C, et al. Abdominal muscle EMG-activity during bridge exercises on stable and unstable surfaces. Phys Ther Sport 2014;15(3):162-8.

27. McGill SM, Karpowicz A. Exercises for spine stabilization: motion/motor patterns, stability progressions, and clinical technique. Arch Phys Med Rehabil 2009;90(1):118-26.

28. Bergmark A. Stability of the lumbar spine. A study in mechanical engineering. Acta Orthop Scand Suppl 1989;230:1-54.

29. Tippett S. Therapeutic exercise for spinal segmental stabilization in low back pain: scientific basis and clinical approach. J Sport Rehabil 2003;12(4):370-1.

30. Barker KL, Shamley DR, Jackson D. Changes in the cross-sectional area of multifidus and psoas in patients with unilateral back pain: the relationship to pain and disability. Spine (Phila Pa 1976) 2004;29(22):E515-9.

31. Danneels LA, Vanderstraeten GG, Cambier DC, Witvrouw EE, De Cuyper HJ. CT imaging of trunk muscles in chronic low back pain patients and healthy control subjects. Eur Spine J 2000;9(4):266-72.

32. Hodges P, Holm AK, Hansson T, Holm S. Rapid atrophy of the lumbar multifidus follows experimental disc or nerve root injury. Spine (Phila Pa 1976) 2006;31(25):2926-33.

33. McGill SM, Grenier S, Kavcic N, Cholewicki J. Coordination of muscle activity to assure stability of the lumbar spine. J Electromyogr Kinesiol 2003;13(4):353-9.

34. O'Sullivan PB. Lumbar segmental 'instability': clinical presentation and specific stabilizing exercise management. Man Ther 2000;5(1):2-12.

35. Arokoski JP, Valta T, Kankaanpää M, Airaksinen O. Activation of lumbar paraspinal and abdominal muscles during therapeutic exercises in chronic low back pain patients. Arch Phys Med Rehabil 2004;85(5):823-32

36. Ferreira-Valente MA, Pais-Ribeiro JL, Jensen MP. Validity of four pain intensity rating scales. Pain 2011;152(10):2399- 
404.

37. Roach KE, Brown MD, Dunigan KM, Kusek CL, Walas M. Testretest reliability of patient reports of low back pain. J Orthop Sports Phys Ther 1997;26(5):253-9.

38. Yates M, Shastri-Hurst N. The Oswestry Disability Index. Occup Med 2017;67(3):241-2.

39. Criswell E. Cram's introduction to surface electromyography. 2nd ed. Sudbury (MA): Jones \& Bartlett; 2011;337-51.

40. Kendall FP, McCreary EK, Provance PG, Rodgers MM, Romani WA. Muscles: testing and function with posture and pain. 5th ed. Baltimore (MD): Lippincott Williams \& Wilkins; 2005;176201.

41. Vera-Garcia FJ, Moreside JM, McGill SM. MVC techniques to normalize trunk muscle EMG in healthy women. J Electromyogr Kinesiol 2010;20(1):10-6.

42. de Oliveira AS, de Morais Carvalho M, de Brum DP. Activation of the shoulder and arm muscles during axial load exercises on a stable base of support and on a medicine ball. J Electromyogr Kinesiol 2008;18(3):472-9.

43. Vezina MJ, Hubley-Kozey CL. Muscle activation in therapeutic exercises to improve trunk stability. Arch Phys Med Rehabil
2000;81(10):1370-9.

44. Suehiro T, Mizutani M, Ishida H, Kobara K, Osaka H, Watanabe S. Individuals with chronic low back pain demonstrate delayed onset of the back muscle activity during prone hip extension. J Electromyogr Kinesiol 2015;25(4):675-80.

45. Yoon TL, Cynn HS, Choi SA, Choi WJ, Jeong HJ, Lee JH, et al. Trunk muscle activation during different quadruped stabilization exercises in individuals with chronic low back pain. Physiother Res Int 2015;20(2):126-32.

46. Masaki M, Tateuchi H, Tsukagoshi R, Ibuki S, Ichihashi N. Electromyographic analysis of training to selectively strengthen the lumbar multifidus muscle: effects of different lifting directions and weight loading of the extremities during quadruped upper and lower extremity lifts. J Manipulative Physiol Ther 2015;38(2):138-44.

47. Marshall PW, Murphy BA. Core stability exercises on and off a Swiss ball. Arch Phys Med Rehabil 2005;86(2):242-9.

48. Kim CR, Park DK, Lee ST, Ryu JS. Electromyographic changes in trunk muscles during graded lumbar stabilization exercises. PM R 2016;8(10):979-89. 